\title{
Simulation de la diffusion des gaz dans les capillaires en régime de Knudsen
}

\author{
G. Aryanpour' ${ }^{1}$ F. Arianpour ${ }^{2}$, M.H. Abbasi ${ }^{1}$ et A. Ershadi ${ }^{3}$ \\ 1 Department of Materials Engineering, Isfahan University of Technology, 84 156, Isfahan, Iran \\ 2 Department of Chemistry, Shahrood University of Technology, Shahrood, Iran \\ 3 Department of Chemistry, Azad University of Shahreza, Shahreza, Iran \\ e-mail : aryanmat@cc.iut.ac.ir - faryan61@yahoo.com - abbasi32@cc.iut.ac.ir - ershadiali@yahoo.com
}

\begin{abstract}
Résumé - Le traitement théorique du transfert de gaz parfait sous un gradient de pression et en régime de Knudsen a abouti à la définition d'une quantité appelée « fraction des particules transmises ». Cette quantité définit la partie diffusée des particules par rapport au nombre total de particules qui entrent dans la voie de diffusion. Un capillaire défini par un tube cylindrique dont l'axe central est perpendiculaire à l'entrée ou à la sortie a été considéré comme la voie de diffusion (Configuration 1). Puis, la diffusion d'un gaz parfait en régime de Knudsen dans un tel tube a été simulée par une méthode de Monte-Carlo. La validité de la simulation a été déduite de la proximité des valeurs théoriques de fraction transmise avec celles obtenues par la simulation. Suite à cette validation, la diffusion de gaz parfait en régime de Knudsen a été simulée dans un tube cylindrique à sections d'entrée et de sortie obliques (Configuration 2). A partir des résultats de la simulation pour la configuration 2, le facteur de tortuosité pour cette configuration a été obtenu.
\end{abstract}

\begin{abstract}
Simulation of Gas Diffusion in Capillaries in Knudsen's Regime - Consideration of ideal gas diffusion under a pressure gradient and in Knudsen regime conducted to the definition of a parameter called the transported gas fraction. This fraction defines the ratio of the diffused particles to the total amount of gas entering into the diffusion path. A capillary defined by a cylindrical tube whose axis is perpendicular to the inlet or outlet (Configuration 1) was considered as the diffusion path and then the computer simulation of gas diffusion in Knudsen regime was carried out by a Monte-Carlo method. Good agreement between simulation and theoretical formulation for the transported gas fraction confirms the validity of the simulation. Following this agreement, the Knudsen diffusion in the cylindrical tube with oblique inlet or outlet (Configuration 2) was simulated. Using the simulation results for the configuration 2, the tortuosity factor for this configuration was obtained.
\end{abstract}




\section{LISTE DES SYMBOLES}

C concentration de gaz $\left(\frac{\text { particule }}{m^{3}}\right)$

$\begin{array}{ll}D_{K} & \text { coefficient de la diffusion de Knudsen } \\ f & \text { fraction des particules diffusées }\end{array}$

$J \quad$ flux de gaz $\left(\frac{\text { particule }}{m^{2} \cdot s}\right)$

$K_{0} \quad$ paramètre caractéristique de voie de diffusion (m)

$k \quad$ Boltzmann's constant $\left(\frac{J}{K}\right)$

$L \quad$ distance horizontale parallèle au gradient de pression macroscopique $(\mathrm{m})$

$m \quad$ masse de particule $(\mathrm{kg})$

$P \quad$ pression de gaz $\left(\frac{N}{m^{2}}\right)$

$p \quad$ nombre des particules diffusées

$q \quad$ nombre des particules non diffusées

$r \quad$ rayon de tube $(\mathrm{m})$

$T$ température absolue (K)

$\begin{array}{ll}\bar{v} & \text { vitesse moyenne des particules de gaz }\left(\frac{m}{s}\right) \\ x, y, z & \text { repère orthogonal }\end{array}$

$\tau \quad$ facteur de tortuosité

$\omega \quad$ angle définissant la configuration 2

\section{INTRODUCTION}

La diffusion des gaz dans un milieu poreux est une étape majeure de plusieurs processus chimiques comme la réduction des minéraux oxydes par des gaz, les réactions hétérogènes, la séparation des différents isotopes d'un élément, etc. [1]. Il est donc important d'avoir une prévision de la quantité de gaz diffusé en milieu poreux qui soit une fonction non seulement des paramètres de gaz mais aussi de la structure poreuse. En fait, selon les paramètres du gaz et de la structure poreuse, on peut diviser le transfert de gaz en deux catégories importants. Il s'agit de la diffusion en régime de Knudsen et en régime ordinaire. En régime de Knudsen, le transfert de gaz est contrôlé par les collisions successives des particules avec la paroi de la voie de diffusion. Autrement dit, le phénomène des collisions des particules les unes avec les autres n'est pas le processus dominant dans le transfert de gaz. En revanche, le régime de transfert ordinaire s'établit quand les particules de gaz se déplacent essentiellement par les nombreuses collisions entre elles-mêmes et non par leurs collisions avec la paroi de la voie de diffusion. Si le rapport du rayon de la voie au libre parcours moyen des particules est inférieur à 0,1 , le mécanisme de transfert de Knudsen est dominant. Dans le cas où ce rapport est supérieur à 10 , c'est le mécanisme ordinaire, qui prévaut. Pour les valeurs de ce rapport comprises entre 0,1 et 10 , les deux mécanismes contrôlent le transfert de gaz [2].
L'étude de la diffusion des gaz dans les milieux poreux, n'est pas très simple pour deux raisons. D'abord le mouvement des particules a une nature complètement aléatoire et deuxièmement la structure poreuse est très complexe. Le développement des ordinateurs à forte puissance et la possibilité de substitution de la structure poreuse par des modèles simplifiés ont permis la simulation de ce phénomène par ordinateur [3-6]. Récemment, une simulation de la diffusion en régime ordinaire dans les tubes cylindriques à différentes configurations a été réalisé dont les résultats ont été comparés avec les résultats théoriques [7,8]. Dans le travail actuel, la diffusion en régime de Knudsen et la procédure de simulation sont expliquées en considérant un tube cylindrique comme la voie de diffusion. Ensuite, les résultats de simulation pour les tubes à différentes configurations sont présentés et discutés.

\section{DIFFUSION DE GAZ EN RÉGIME DE KNUDSEN}

\subsection{Tube cylindrique de la configuration 1}

La figure 1 montre un gradient de la pression du gaz parfait dans un tube cylindrique. Le gradient de pression est sur la direction horizontale $z$ qui est parallèle à l'axe central du tube. Autrement dit, l'entrée ou la sortie du tube est perpendiculaire à l'axe du tube. Désormais, nous qualifierons cette configuration de « configuration 1 ». Le gaz se déplace sous l'effet du gradient de pression c'est-à-dire de la pression $P_{1}$ à la pression nulle. On peut considérer que les particules se déplacent essentiellement par leurs collisions successives avec la paroi du tube. Autrement dit, la particule entre en collision avec la paroi du tube avant de parcourir son libre parcours moyen complet. Après la collision, la particule est absorbée sur la surface de la paroi et réfléchie avec un angle aléatoire de la paroi. Nous pouvons imaginer l'établissement de ces conditions dans un domaine de pression très basse en considérant un tube donné ou dans le cas d'un tube à rayon très petit devant le libre parcours moyen des particules.

Le transfert de gaz sous un tel gradient de pression et par le mécanisme de collisions avec la paroi peut être formulé par la première loi de Fick :

$$
J=-D_{K} \frac{d C}{d z}
$$

$J$ est le flux de diffusion décrivant le nombre des particules traversant la section unitaire perpendiculaire à l'axe $z$ pendant l'unité de temps. $D_{K}$ est le coefficient de diffusion de Knudsen et $C$ est la concentration de gaz dans le tube en fonction de $z$. On a déjà défini $D_{K}$ par la fonction suivante [9] :

$$
D_{K}=\frac{4}{3} \bar{v} K_{0}
$$




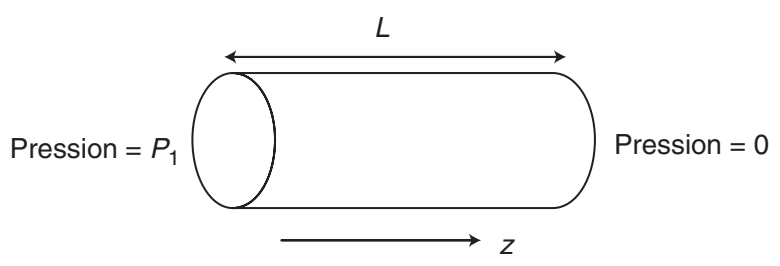

Figure 1

Illustration schématique d'une porosité cylindrique dans laquelle un gaz parfait est transféré sous son gradient horizontal de pression.

Schematical illustration of a cylindrical pore in which an ideal gas is transferred under a horizontal pressure gradient.

où $\bar{v}$ est la vitesse moyenne des particules et $K_{0}$ avec la dimension d'une longueur, est un paramètre caractéristique du milieu dans lequel le gaz se transfère. Dans le cas d'un tube dont la demi-longueur $\left(L^{\prime}\right)$ est beaucoup plus grande que son rayon $(r)$ i.e. $L^{\prime}>>r$, et dans les conditions où le libre parcours moyen des particules est très grand (tend vers l'infini), le coefficient de diffusion suivant est proposé [10] :

$$
D_{K}=\frac{2}{3} \bar{v} r\left(1-\frac{3 r}{4 L^{\prime}}\right) \quad, \quad L^{\prime}>>r
$$

Dans le cas d'un tube de longueur infinie, nous aurons :

$$
D_{K}=\frac{2}{3} \bar{v} r \quad, \quad L^{\prime} \rightarrow \infty
$$

Cette formulation équivaut à celle présentée par Knudsen pour la première fois [11]. Ainsi :

$$
\begin{gathered}
K_{0}=\frac{r}{2}\left(1-\frac{3 r}{2 L}\right) \quad, \quad L>>r \\
K_{0}=\frac{r}{2} \quad, \quad L \rightarrow \infty
\end{gathered}
$$

où $L$ est la longueur du tube (fig. 1). $\bar{v}$ dans l'équation 2 est écrit sous la forme suivante [12] :

$$
\bar{v}=\left(\frac{8 k T}{\pi m}\right)^{\frac{1}{2}}
$$

où $k, m$ et $T$ sont la constante de Boltzmann, la masse de la particule et la température absolue, respectivement. En appliquant la loi des gaz parfaits, l'équation 1 peut être remplacée par :

$$
J=-\frac{4}{3}\left(\frac{8 k T}{\pi m}\right)^{\frac{1}{2}} K_{0} \frac{d P}{d z} \frac{1}{k T}
$$

En régime permanent, $J$ est constant ce qui prouve l'existence d'un gradient linéaire de pression suivant l'axe $z$. Nous aurons donc :

$$
J=\frac{4}{3}\left(\frac{8 k T}{\pi m}\right)^{\frac{1}{2}} K_{0} \frac{P_{1}}{L} \frac{1}{k T}
$$

Il est évident qu'en raison de la nature aléatoire de ce transfert, toutes les particules qui entrent dans le tube de la pression $P_{1}$ (flux entrant) ne sont pas capables de parcourir toute la longueur du tube. Autrement dit, le flux effectif $J$ est une fraction $(f)$ du flux entrant. Ce dernier est déterminé d'après la théorie cinétique des gaz par $P_{1}(2 \pi m k T)^{\frac{-1}{2}}$. Nous aurons donc :

$$
J=f P_{1}(2 \pi m k T)^{\frac{-1}{2}}
$$

Alors :

$$
-\frac{4}{3}\left(\frac{8 k T}{\pi m}\right)^{\frac{1}{2}} K_{0} \frac{-P_{1}}{L} \frac{1}{k T}=f P_{1}(2 \pi m k T)^{\frac{-1}{2}}
$$

$K_{0}$ est donc égale à :

$$
K_{0}=\frac{3 L f}{16}
$$

Si on applique les équations 5 et/ou 6 dans l'équation 12, nous aurons respectivement :

$$
\begin{gathered}
f=\frac{8}{3}\left(\frac{r}{L}\right)-4\left(\frac{r}{L}\right)^{2}, \quad L>>r \\
f=\frac{8}{3}\left(\frac{r}{L}\right), \quad L \rightarrow \infty
\end{gathered}
$$

On appelle désormais $f$ la fraction des particules transmises ou diffusées. Ce traitement permet donc d'obtenir $K_{0}$ par $f$ et ensuite $D_{K}$ en appliquant l'équation 2. Ainsi, le flux de gaz dans le milieu considéré sera calculable. L'idée de trouver le paramètre $f$ par la simulation numérique est donc exploitable.

\subsection{Tube cylindrique de la configuration 2}

Considérons encore le transfert du gaz parfait en régime de Knudsen dans un milieu poreux avec des tubes cylindriques. Ce transfert est provoqué par le gradient horizontal de pression sur la distance $L$ parallèle à l'axe $z$ (fig. 2). A part le tube de la configuration 1 montré sur la figure 2 , nous pouvons considérer un autre tube cylindrique comme voie de transfert. Ce tube est distingué cette fois par ses sections d'entrée et de sortie obliques. Autrement dit, les sections d'entrée et de sortie ne sont plus à angle droit avec l'axe du tube et les 
particules, pour un parcours horizontal effective de longueur $L$, doivent parcourir une distance supérieure à $L$. Désormais, nous qualifierons cette configuration de « configuration 2 ». Cette configuration peut être caractérisée par l'angle $\omega$ défini entre l'axe central du tube et l'axe $z$. Cet angle est présenté dans la figure 2 et il caractérise l'écart entre les deux configurations, sachant que $\omega=0$ définit la configuration 1 . La configuration 2 nous intéresse car dans l'étude des milieux poreux et des phénomènes voisins, il est très courant de remplacer le milieu poreux par un modèle plus simple. L'un de ces modèles est par exemple le modèle avec connexion des tubes cylindriques aux deux configurations [13]. Ceci explique le choix de la configuration 2 pour la suite de cette étude.

Comme mentionné précédemment, pour une diffusion effective sur une distance $L$ parallèle à l'axe $z$, mais dans le tube de la configuration 2 , les particules doivent parcourir une distance supérieure à $L$. Alors, le passage de la configuration 1 à la configuration 2 est équivalent à un allongement du tube de la configuration 1. La question importante est comment traiter mathématiquement le transfert des particules dans la configuration 2 ? Il est évident que les équations 1 et 2 considèrent le cas d'un tube horizontal pour lequel le gradient horizontal de pression est déterminé sur sa longueur réelle tandis que dans la configuration 2, la distance horizontale et effective sur laquelle le gradient de pression est établi $(L)$ est différente de la longueur réelle de la configuration 2. Alors, en considérant toujours le même gradient horizontal de pression dans l'équation 1, nous devons appliquer un coefficient de diffusion inférieur pour le cas de la configuration 2 en raison de l'allongement du parcours par rapport à la configuration 1 . Un coefficient de diffusion inférieur est déterminé en considérant un facteur $\tau$ appelé le facteur de tortuosité. Ainsi, le coefficient de diffusion donné par l'équation 2 se transforme suivant l'équation ci-dessous pour le cas de la configuration 2 [9] :

$$
D_{K, C 2}=\frac{1}{\tau} \frac{4}{3} \bar{v} K_{0}
$$

où $D_{K, C 2}$ est le coefficient de diffusion dans la configuration 2. Le facteur de tortuosité $(\tau)$ est évidemment supérieur à 1 . Il faut remarquer que l'équation (15) s'écrit en considérant un tube de la configuration 2 avec le même rayon que le tube de la configuration 1. Si on note $\frac{K_{0}}{\tau}$ dans l'équation 15 comme
$K_{0, \mathrm{C} 2}$, on peut écrire :

$$
D_{K, C 2}=\frac{4}{3} \bar{v} K_{0, C 2}
$$

On peut également appliquer la démarche à partir de l'équation 3 jusqu'à l'équation 8 afin d'obtenir la fraction des particules transmises dans la configuration 2 que l'on appelle $f^{\prime}$ :

$$
K_{0, C 2}=\frac{3 L f^{\prime}}{16}
$$

Tube de la configuration 2

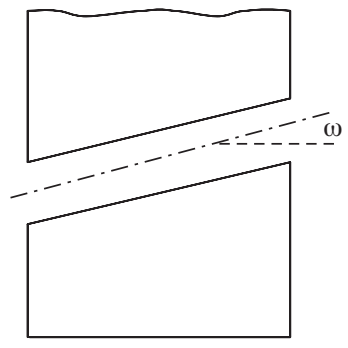

Tube de la configuration 1

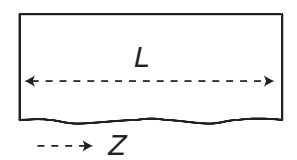

Figure 2

Tubes cylindriques des deux configurations en deux dimensions.

Two configurations of cylindrical tube shown in two dimensions.

ou :

$$
\frac{K_{0}}{\tau}=\frac{3 L f^{\prime}}{16}
$$

ou :

$$
K_{0}=\frac{3 \tau L f^{\prime}}{16}
$$

La comparaison des équations 12 et 19 donne :

$$
\frac{f}{f^{\prime}}=\tau
$$

Ainsi, $f$ et $f^{\prime}$ obtenus par la simulation peuvent être utilisés pour déterminer le facteur de tortuosité de la configuration 2. Il faut remarquer que $f$ est identique à $f^{\prime}$ à $\omega=0$ i.e. $f^{\prime} \omega=0$.

\section{PROCÉDURE DE SIMULATION}

Il est évident que lors du transfert de gaz dans une voie soumise à un gradient de pression (de $P_{1}$ à zéro), des particules entrent en grand nombre sous la pression $P_{1}$ et se déplacent simultanément dans la voie (fig. 3). Comme on l'a dit précédemment, les particules se déplacent par les collisions aléatoires avec la paroi en régime de Knudsen. Alors, on peut imaginer qu'une partie des particules réussit à sortir du tube et le reste, en franchissant partiellement la longueur du tube, puis en sortant par l'entrée du tube, retournent à la source de pression. La fraction des particules transmises est en fait le rapport de la partie diffusée au nombre total des particules entrant dans le tube. Il est évident que la simulation par ordinateur n'impose pas de décrire simultanément le 
mouvement d'un grand nombre de particules. Par contre, il est possible d'élaborer un programme informatique décrivant le déplacement d'une seule particule dans la voie considérée et d'exécuter ce programme plusieurs fois. Ainsi, à chaque exécution, le déplacement d'une particule est simulé. La fraction des particules transmises est donc calculable après un grand nombre d'exécutions du programme informatique.

Après cette introduction, il faudrait expliquer le programme et la procédure de simulation. Comme mentionné précédemment, nous devons définir tout d'abord la voie de diffusion. Dans ce travail, deux tubes cylindriques aux configurations 1 et 2 sont considérés comme les voies de diffusion.

\subsection{Simulation pour le tube de la configuration 1}

Considérons la diffusion d'un gaz parfait pur sous un gradient de pression entre $P_{1}$ et 0 dans le tube de la configuration 1 avec rayon $r$ et longueur $L$ allongé parallèle à l'axe $z$ (fig. 3). Supposons l'entrée du tube est placée à une coordonnée $z$, par exemple $z=d$. Dans la suite, l'entrée d'une particule de la pression $P_{1}$ à l'intérieur du tube ainsi que son mouvement dans ce tube par les collisions successives avec la paroi seront décrits.

La figure 4 illustre l'entrée de la particule à l'intérieur du tube. Pour cela, un point $\mathrm{N}$ est sélectionné d'une façon aléatoire sur la surface circulaire de l'entrée. Ainsi, le point $\mathrm{E}$ est situé sur le plan $x y$ aux mêmes coordonnées $x$ et $y$ que le point $\mathrm{N}$. Si on choisit encore d'une manière aléatoire le point M placé sur le plan $x y$, on peut dire que la direction aléatoire $\overrightarrow{\mathrm{MN}}$ définit la direction de l'entrée de particule dans le tube. Pour déterminer aléatoirement le point $\mathrm{M}$, deux angles aléatoires $\theta$ et $\beta$ sont sélectionnés. L'angle $\theta$, une valeur à probabilité égale entre 0 et $\frac{\pi}{2}$, définit l'angle entre $\overrightarrow{\mathrm{NE}}$ et $\overrightarrow{\mathrm{NF}}$ (F est placé sur le plan $x y$ à la même coordonnée $x$ que $\mathrm{N}$ ou E). $\beta$, une valeur encore à probabilité égale entre 0 et $2 \pi$, définit donc la rotation de $\mathrm{F}$ autour de $\mathrm{E}$ dans le plan $x y$. Ainsi, deux points de la direction $\overrightarrow{\mathrm{MN}}$ ont les coordonnées suivantes :

$$
\mathrm{N}\left\{\begin{array} { l } 
{ x _ { \mathrm { N } } } \\
{ y _ { \mathrm { N } } } \\
{ d }
\end{array} \quad \text { et } \quad \mathrm { M } \left\{\begin{array}{l}
x_{\mathrm{N}}+(d \tan \theta \cdot \sin \beta) \\
y_{\mathrm{N}}+(d \tan \theta \cdot \cos \beta) \\
0
\end{array}\right.\right.
$$

Le vecteur $\overrightarrow{\mathrm{MN}}$, direction aléatoire de l'entrée de particule, est donc connu. Il sera donc possible de trouver le point d'intersection de $\overrightarrow{\mathrm{MN}}$ avec le tube. Maintenant, considérons que le point d'intersection est déterminé et que la particule doit se séparer de la paroi encore d'une manière aléatoire. Considérons la figure 5 pour formuler cette étape. Le dernier point d'intersection de la particule est montré par le point $\mathrm{V}$ sur cette figure. Les coordonnées du point $\mathrm{V}$ sont $x_{v}, y_{v}$, et $z_{v}$.

Les points ci-dessous sont nécessaires pour définir la direction aléatoire de la séparation de particule $(\overrightarrow{\mathrm{VW}})$.

- Deux rayons perpendiculaires de la section circulaire du tube sont choisis comme les vecteurs $\overrightarrow{S_{1}}$ et $\overrightarrow{S_{2}}$. Comme la figure 5 le montre, $\overrightarrow{S_{1}}$ passe sur V. En plus, $\overrightarrow{S_{2}}$ est défini de façon que l'observateur allongé sur $\overrightarrow{S_{1}}$ qui regarde la fin du vecteur $\overrightarrow{S_{2}}$, doive voir à l'intérieur du tube en tournant dans le sens des aiguilles d'une montre. Il faut noter que $\overrightarrow{S_{2}}$ se trouve dans le plan $\mathrm{Q}$ normal à $\overrightarrow{S_{1}}$.

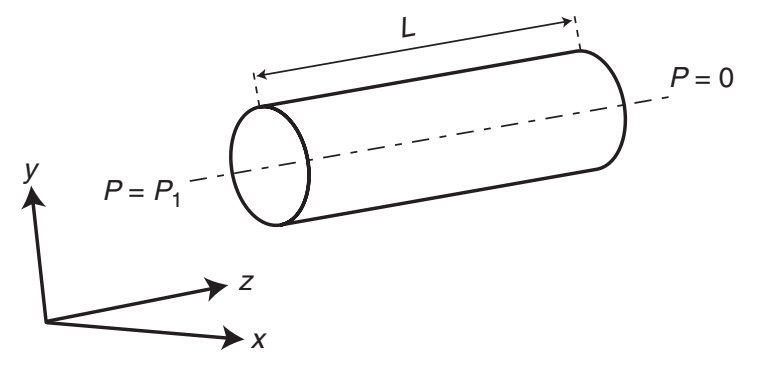

Figure 3

Le repère trigonal avec le tube allongé parallèle à l'axe $z$. Cartesian coordinates with a cylindrical tube parallel to the $z$ axis.

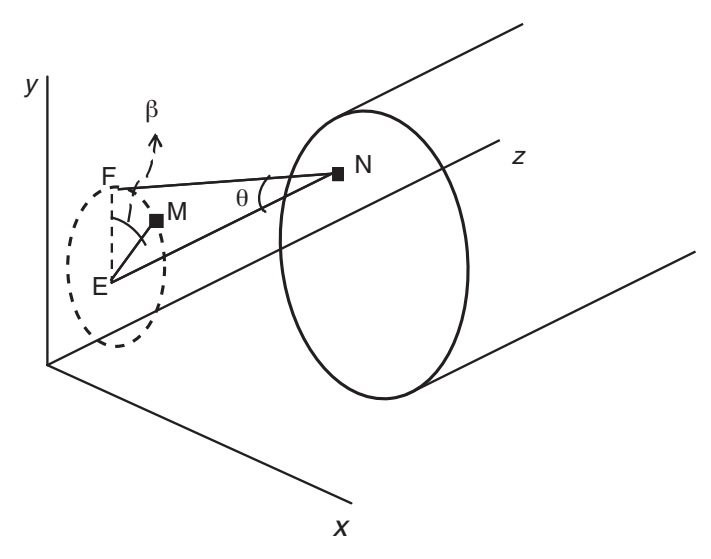

Figure 4

Illustration de l'entrée d'une particule dans le tube avec une direction aléatoire $\overrightarrow{\mathrm{MN}}$.

Illustration of particle entrance into the tube with a random direction $\overrightarrow{\mathrm{MN}}$. 


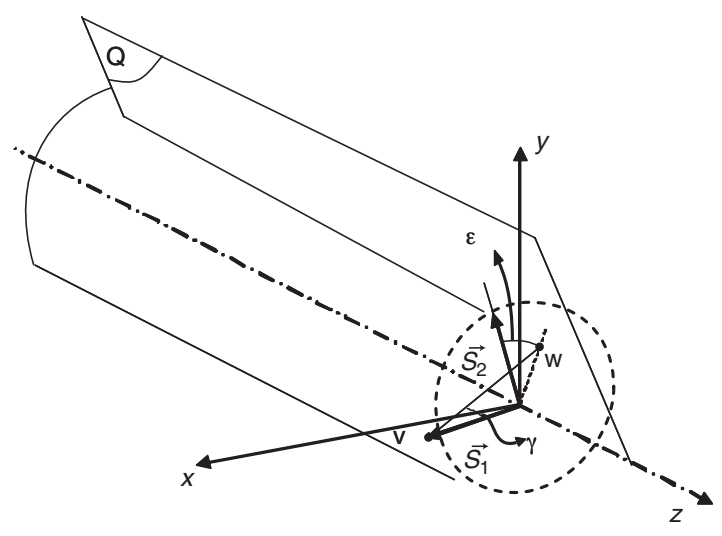

Figure 5

Choix de la direction aléatoire de la séparation de particule $(\overrightarrow{\mathrm{VW}})$ située au point $\mathrm{V}$ de la paroi.

Selection of a random direction $(\overrightarrow{\mathrm{VW}})$ for the separation of the particle located at point $V$ of the tube wall.

- Deux angles $\gamma$ et $\varepsilon$ sont définis d'une façon aléatoire. $\gamma$ est compris entre 0 et $\frac{\pi}{2}$. La probabilité de cet angle suit une fonction en cosinus à cause de l'existence des rugosités même à l'échelle microscopique [9]. Par $\varepsilon$, une valeur aléatoire à probabilité égale entre 0 et $2 \pi$ est choisie. Ces angles sont représentés sur la figure 5 . On remarque que par $\gamma$ une surface conique avec l'axe $\overrightarrow{S_{1}}$ est définie et qu'une génératrice de cette surface est choisie par $\varepsilon$.

Ainsi, les coordonnées du point W dans le plan Q (Q contient $\overline{S_{2}}$ et est perpendiculaire à $\overrightarrow{S_{1}}$ ) sont :

$\mathrm{W} \mid \begin{aligned} & x_{\mathrm{C}}-r \tan \gamma \cdot \cos \varepsilon .\left(\text { cosinus de l'angle de } \overrightarrow{S_{1}} \text { avec l'axe } y\right) \\ & y_{\mathrm{C}}+r \tan \gamma \cdot \cos \varepsilon .\left(\text { cosinus de l' angle de } \overrightarrow{S_{1}} \text { avec l'axe } x\right) \\ & z_{\mathrm{V}}-r \tan \gamma \cdot \sin \varepsilon\end{aligned}$

où, $x_{c}, y_{c}$ sont les coordonnées $x$ et $y$ du centre de tube, respectivement. Connaissant les coordonnées du point $\mathrm{V}$, on peut obtenir l'équation de $\overrightarrow{\mathrm{VW}}$ pour déterminer le nouveau point d'intersection de la particule avec la paroi du tube. Il est évident que ce calcul peut être répété pour trouver les points successifs d'intersection. À la fin de chaque calcul, on peut vérifier si le calcul doit se répéter dans le cas où la coordonnée $z$ de l'intersection est comprise entre $d$ et $(L+d)$ où $L$ est la longueur du tube, ou si le calcul doit s'arrêter pour la particule considérée quand cette coordonnée est inférieure à $d$ ou supérieure à $(L+d)$. Ainsi, nous pouvons simuler le mouvement d'un grand nombre de particules par une exécution volumineuse du programme informatique. Enfin, nous aurons deux nombres, l'un est le nombre des particules ayant pu diffuser sur toute la longueur du tube $(p)$ et le nombre des particules qui sont retournées à la source de pression après avoir partiellement diffusé dans le tube $(q)$. Il est évident que la fraction des particules transmises est calcu par :

$$
f=\frac{p}{p+q}
$$

\subsection{Simulation pour le tube de la configuration 2}

Les principes respectés pour la configuration 1 ont été développés pour simuler le mouvement des particules dans le tube de la configuration 2. Ainsi, le facteur $f^{\prime}$ est calculé.

\section{RÉSULTATS ET DISCUSSION}

\subsection{Configuration 1}

Dans cette partie, nous allons présenter les résultats de la simulation pour le tube de la configuration 1 . Il faut remarquer qu'à chaque simulation, le déplacement d'un nombre total des particules égale à 100000 a été simulé. Le résultat de la répétition du programme est relativement stable pour ce nombre de particules, voire pour un nombre inférieur. Ce point est illustré sur la figure 6 pour une valeur arbitraire de $r / L$ égale à 0,01 . On constate que la différence entre les valeurs maximum et minimum de $f$ obtenues par six exécutions du programme, peut être ignorée pour un nombre total de particules égale à 100000 .

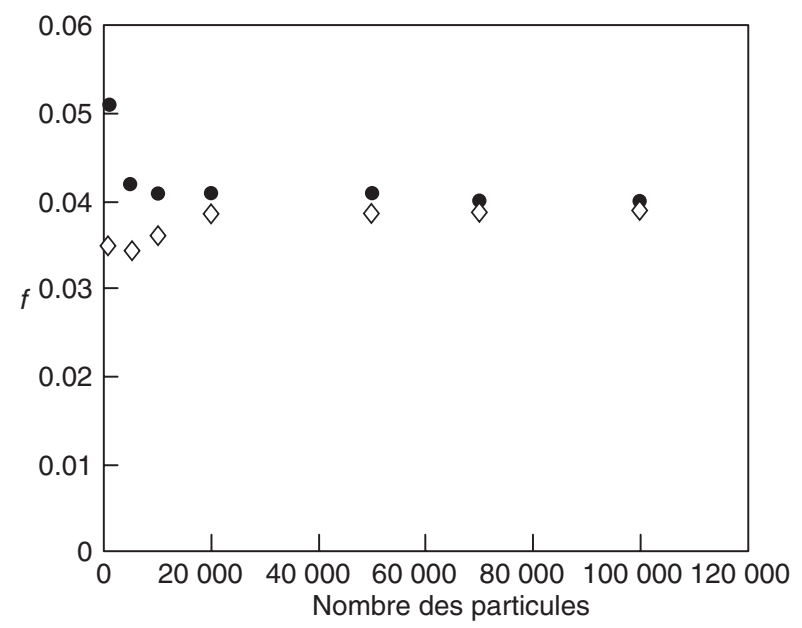

Figure 6

Convergence des résultats de simulation quand le nombre total des particules utilisé pour la simulation tend vers 100000 . Les cercles et les carrés montrent les valeurs maximum et minimum de $f$ respectivement, obtenues par six exécutions du programme.

Convergence of the simulation results when the total particle number used for simulation tends toward 100000 . Circles and squares show the max. and min. values of $f$ respectively obtained from six runs of the program. 
Le choix du nombre des particules modélisées permet par la suite de lancer les simulations. Les résultats obtenus en accord avec la formulation présentée, sont fonctions seulement de $(r / L)$. Ainsi, dans la figure 7, le paramètre $f$ provenant de la simulation est représenté par des cercles en fonction de $(r / L)$. La courbe en trait plein résulte de l'application de l'équation 13. Comme cette équation est de degré deux par rapport à $(r / \mathrm{L})$, elle diminue après son point de maximum à $r / L=1 / 3$. Il faut remarquer que cette équation est valide dans le domaine des $r / L$ très petits. En conséquence, il se peut qu'on ne puisse pas qualifier de positive la proximité des deux résultats dans le domaine de grand $(r / L)$, proche de $r / L=1 / 3$, car le mouvement des particules ne serait pas contrôlé par les collisions successives des particules avec la paroi dans ce domaine. Le plus important est la proximité des deux résultats aux petites valeurs de $(r / L)$. Nous pouvons constater la validité des résultats dans le domaine de $(r / L)$ inférieur à 0,03 où le nombre moyen de collisions par particule est élevé. Le nombre moyen de collisions par particule, illustré par la figure 8, est calculé en divisant le nombre total des collisions avec la paroi par le nombre total de particules considérées pour chaque exécution.

\subsection{Configuration 2}

Dans cette partie, les résultats de la simulation pour un tube de la configuration 2 sont analysés. Nous nous intéressons à la variation de la fraction des particules transmises $\left(f^{\prime}\right)$ ainsi qu'au facteur de tortuosité en fonction de l'angle $\omega$. Le tableau 1 synthétise ces variations pour une valeur arbitraire de $r / L$ égale à 0,01 . Il faudrait préciser que la valeur de $\tau$ citée dans ce tableau est obtenue par l'application de l'équation 20 dont le paramètre $f$ est la valeur de $f^{\prime}$ à $\omega=0$ $\left(f_{\omega=0}^{\prime}\right)$ i.e. :

$$
\tau=\frac{f_{\omega=0}^{\prime}}{f^{\prime}}
$$

Dans le cas de $r / L=0,01, f_{\omega=0}^{\prime}$ est égale à 0,04027 .

Il faut aussi noter que le nombre total des particules pour obtenir chaque résultat du tableau 1 est de 100 000. En outre, il est très probable que la valeur de $\tau$ dans le domaine de grand $\omega$ ne soit pas très fiable. À partir des résultats présentés dans le tableau 1, il est donc possible d'approcher $\tau(\omega)$ par l'expression suivante :

$$
\tau=\frac{90(0,034877 \omega+1)}{90-\omega}
$$

où $\omega$ est en degré. Les carrés dans la figure 9 sont les valeurs déjà présentées dans le tableau 1 et on peut constater que la courbe obtenue du lissage i.e. l'équation 25 , passe bien sur les carrés. La figure 9 montre aussi les valeurs du facteur de tortuosité obtenues par la simulation pour la configuration 2 à $r / L=0,02$ (les cercles) qui sont logiquement très proches des carrés ou de la courbe de lissage. Si dans une démarche inverse, on met l'équation 25 dans 24 , on peut obtenir l'équation dite de lissage pour $f^{\prime}$ i.e. $f^{\prime}(\omega)$ :

$$
f^{\prime}=\frac{f_{\omega=0}^{\prime}(90-\omega)}{90(0,034877 \omega+1)}
$$

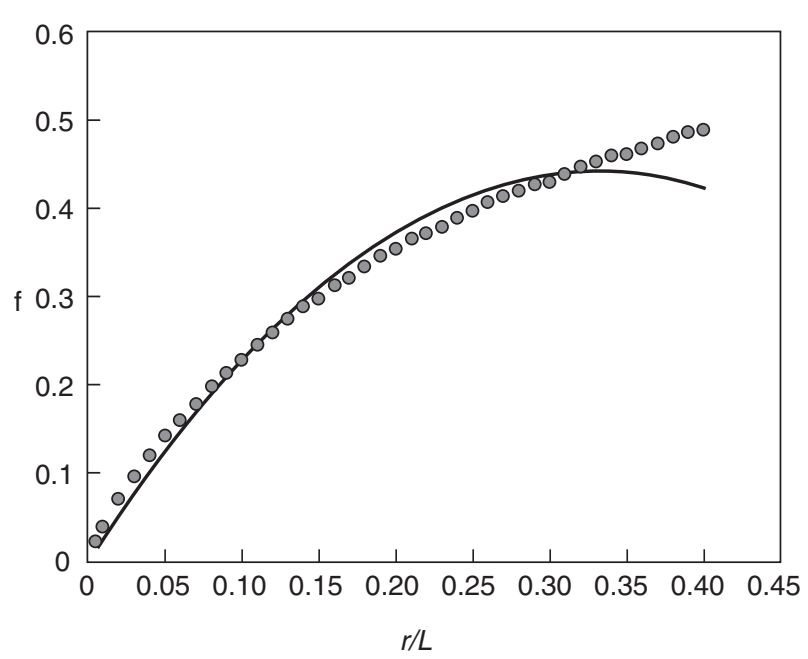

Figure 7

$f$ comme fonction de $r / L$.

Points : les résultats de la simulation. Courbe : l'équation 13.

$f$ as a function of $r / L$.

Points: Simulation results. Solid curve: Equation 13.

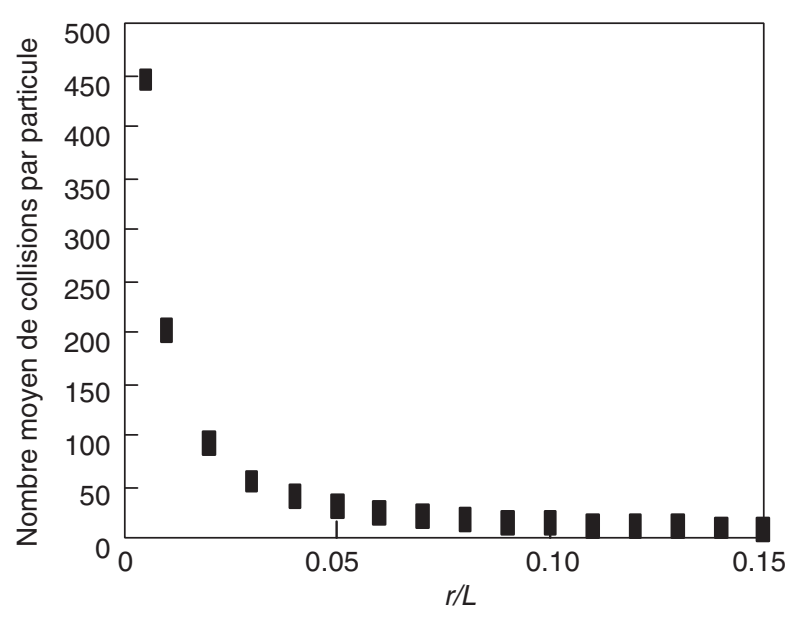

Figure 8

Nombre moyen de collisions avec la paroi obtenu par la simulation.

Average number of particle collisions with the tube wall obtained from simulation. 
TABLEAU 1

Variation de $f^{\prime}$ et de $\tau$ avec l'angle $\omega$

Variation of $f^{\prime}$ and $\tau$ with respect to $\omega$

\begin{tabular}{c|c|c}
\hline$\omega$ (degré) & $f^{\prime}$ (simulation) & $\tau$ \\
\hline 0 & 0,04027 & 1,00 \\
5 & 0,02948 & 1,37 \\
10 & 0,02582 & 1,56 \\
15 & 0,02276 & 1,77 \\
20 & 0,02015 & 2,00 \\
25 & 0,01764 & 2,28 \\
30 & 0,01596 & 2,52 \\
35 & 0,01433 & 2,81 \\
40 & 0,01166 & 3,45 \\
45 & 0,01009 & 3,99 \\
50 & 0,00784 & 5,14 \\
55 & 0,00594 & 6,78 \\
60 & 0,00464 & 8,68 \\
65 & 0,00313 & 12,87 \\
70 & 0,00244 & 16,50 \\
75 & 0,00134 & 30,05 \\
80 & 0,00068 & 59,22 \\
85 & 0,00017 & 236,88 \\
88 & 0,00012 & 335,58 \\
\hline
\end{tabular}

Nous attendons évidemment à ce que la courbe obtenue de la fonction (26) soit très proche des valeurs obtenues de la simulation et présentées dans le tableau 1. La figure 10 confirme bien ce point.
Ainsi, pour un tube avec $r / L$ quelconque, on peut écrire :

$$
f^{\prime}=\frac{f_{\omega=0}^{\prime}(90-\omega)}{90(0,034877 \omega+1)}
$$

\section{CONCLUSION}

Le transfert de gaz parfait pur dans un capillaire défini par un tube cylindrique, a été simulé en considérant le mécanisme de transfert de Knudsen sous un gradient horizontal de pression. Deux configurations pour le tube ont été considérées. La configuration 1 correspond à un tube parallèle au gradient horizontal de pression. La configuration 2 correspond au tube dont les sections d'entrée et de sortie sont obliques. Dans le cas de la configuration 1 , le résultat de la simulation pour un rapport très petit du rayon à la longueur correspond bien au calcul théorique présenté précédemment. La validité de la simulation pour la configuration 1 justifie celle d'un tube de la configuration 2 . Les résultats obtenus à ce stade permettent d'estimer la variation du facteur de tortuosité du tube de la configuration 2 évidemment selon le mécanisme de transfert en régime de Knudsen. Finalement, il est remarquable que ce travail puisse se développer pour la simulation du transfert dans un milieu poreux dont la structure poreuse est définie par un réseau des tubes de la configuration 2.

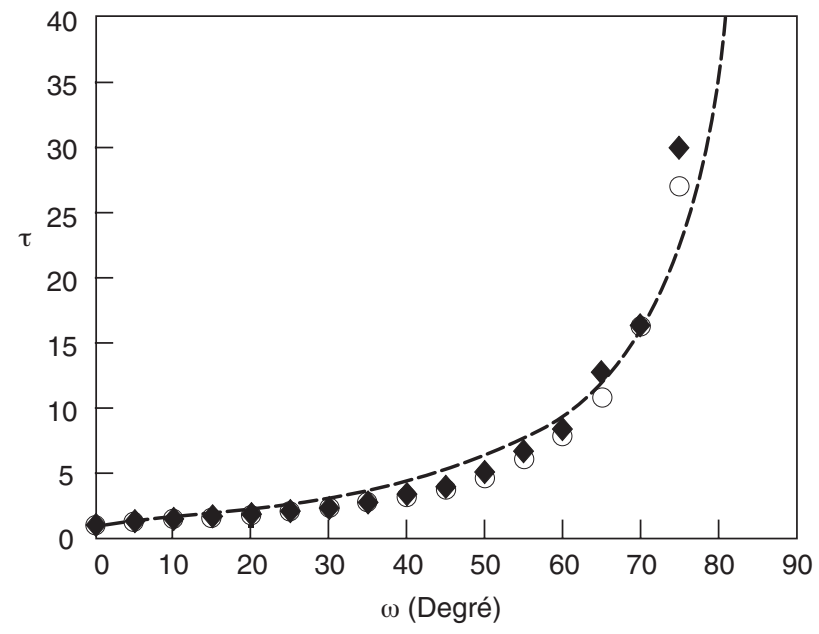

Figure 9

Variation de $\tau$ en fonction de $\omega$. Carrés : $\tau$ cité dans le tableau 1 . Courbe : l'équation 25. Cercles : $\tau$ obtenu par la simulation pour $r / L=0,02$.

Variation of $\tau$ with respect to $\omega$. Squares: $\tau$ given by Table 1 . Curve: Equation 25. Circles: $\tau$ obtained from simulation for $r / L=0.02$.

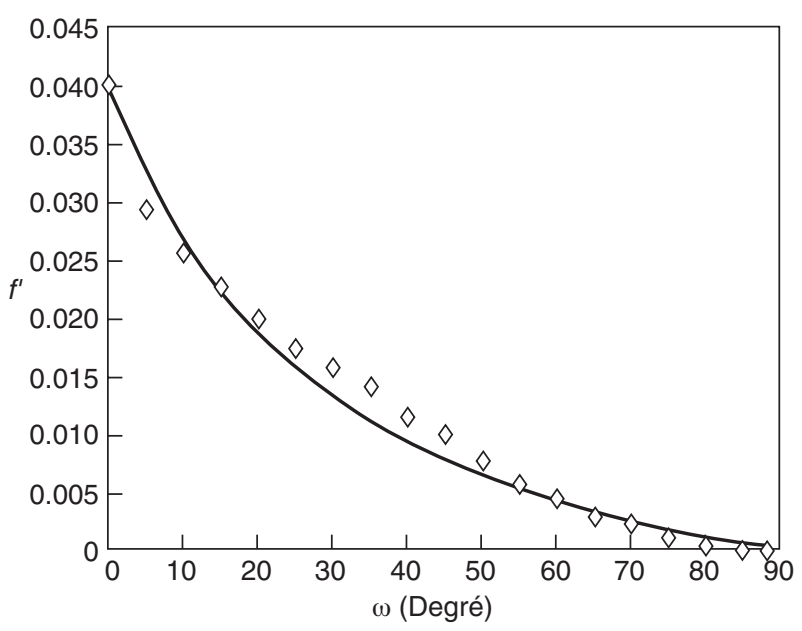

Figure 10

Variation de la fraction des particules transmises pour la configuration 2 en fonction de $\omega(r / L=0,01)$. Carrés : les résultats de la simulation (tableau 1). Courbe : l'équation 26.

Variation of the transported gas fraction for the configuration 2 as a function of $\omega(r / L=0.01)$. Squares: Simulation results (Tab. 1). Curve: Equation 26. 


\section{REFERENCES}

1 Sahimi M., Gavals G.R., Tsotsis T.T. (1990) Statistical and continuum models of fluid-solid reactions in porous media, Chem. Eng. Sci. 45, 1443-1502.

2 Youngquist G.R. (1970) Diffusion and flow of gases in porous solids, Ind. Eng. Chem. 62, 52-63.

3 Abbasi M.H., Evans J.W., Abramson I.S. (1983) Diffusion of gases in porous solids: Monte Carlo simulation in the Knudsen and ordinary diffusion regimes, AIChE J.29, 617-624.

4 Akanni K.A., Evans J.W., Abramson I.S. (1987) Effective transport coefficients in heterogeneous media, Chem. Eng. Sci. 42, 1945-1954.

5 Evans J.W., Abbasi M.H., Sarin A. (1980) A Monte Carlo simulation of the diffusion of gases in porous solids, J. Chem. Phys. 72, 2967-2973.

6 Nakano Y., Evans J.W. (1980) Monte-Carlo simulation of diffusion of gases in porous solid: Calculation for a new class of solids, J. Chem. Phys. 78, 2568-2577.
7 Aryanpour G., Abbasi M.H. (2005) Computer simulation of ordinary gas transfer in tubes, J. Porous Media 8, 379-391.

8 Aryanpour G., Abbasi M.H. (2006) Simulation of ordinary gas transfer in porous solid with tubular inclined pores, J. Porous Media 9, 261-273.

9 Szekely J., Evans J.W., Sohn H.Y. (1976) Gas-Solid Reactions, Academic Press, New York.

10 Pollard W.G., Present R.D. (1948) On gaseous self-diffusion in long capillary tubes, Phys. Rev. 73, 762-774.

11 Knudsen M. (1909) The law of the molecular flow and viscosity of gases moving through tubes, Ann. Phys. 28, 75-130.

12 Levine I.N. (1988) Physical Chemistry, 3rd ed., McGraw-Hill, New York.

13 Petersen E.E. (1957) Reaction of porous solids, AIChE 3, 443448.

Manuscrit définitif reçu en mai 2007

Copyright (c) 2007 Institut français du pétrole

Permission to make digital or hard copies of part or all of this work for personal or classroom use is granted without fee provided that copies are not made or distributed for profit or commercial advantage and that copies bear this notice and the full citation on the first page. Copyrights for components of this work owned by others than IFP must be honored. Abstracting with credit is permitted. To copy otherwise, to republish, to post on servers, or to redistribute to lists, requires prior specific permission and/or a fee: Request permission from Documentation, Institut français du pétrole, fax. +33147527078 , or revueogst@ifp.fr. 